Open Access

\title{
Key components in models of community- based interventions coordinating care in dementia: a mixed studies systematic review protocol
}

Amy Backhouse ${ }^{1,2^{*}}$, Chris Dickens ${ }^{1,2}$, David Richards ${ }^{1,2}$ and Rose McCabe ${ }^{1,2}$

\begin{abstract}
Background: Current health and social care systems are providing suboptimal and fragmented care to the growing dementia population. Interventions aiming to coordinate care services for individuals with dementia and their families are already widely used; however, the structure and implementation of these interventions vary. This mixed studies review aims to investigate the key components of effective community-based interventions that focus on coordinating care in dementia.

Methods: We will search MEDLINE, Cochrane Library, Embase and PsycINFO databases for studies of any design that look at community-based interventions that aim to coordinate dementia care through the allocation of a specified professional responsible for provision of care. Health Management Information Consortium (HMIC), Social Policy and Practice (SPP), ProQuest and International Clinical Trials Registry Platform (ICTRP) databases will be searched for grey literature. Outcomes of interest are health outcome measures that relate to the individual with dementia and/or informal caregiver, measures of resource use or process measures. Two independent reviewers will screen identified papers and extract data from eligible studies. Evidence synthesis will take place in three stages, and methods will be largely dependent on the data available. A sequential review design will be used where the qualitative evidence will be synthesised first, focusing on stakeholder's subjective views of key components. This will drive forward the quantitative stage which will identify key components of effective interventions. The final stage of the review will merge the two strands of evidence through a narrative synthesis.
\end{abstract}

Discussion: The results from this review will be used to develop a model for a community-based intervention coordinating care in dementia. Furthermore, the findings will help guide future work on intervention development of health and social care services for dementia.

Systematic review registration: PROSPERO CRD42015024618

Keywords: Systematic review protocol, Mixed studies review, Synthesis, Dementia, Care management, Collaborative care, Case management, Community interventions, Care coordination, Health services

\footnotetext{
* Correspondence: a.backhouse@exeter.ac.uk

'University of Exeter Medical School, College House, St Luke's Campus, Exeter EX1 2LU, UK

${ }^{2}$ National Institute for Health Research (NIHR) Collaboration for Leadership in Applied Health Research and Care (CLAHRC) South West Peninsula, South Cloisters, St Luke's Campus, Exeter EX1 2LU, UK
}

(c) 2015 Backhouse et al. Open Access This article is distributed under the terms of the Creative Commons Attribution 4.0 International License (http://creativecommons.org/licenses/by/4.0/), which permits unrestricted use, distribution, and reproduction in any medium, provided you give appropriate credit to the original author(s) and the source, provide a link to the Creative Commons license, and indicate if changes were made. The Creative Commons Public Domain Dedication waiver (http://creativecommons.org/publicdomain/zero/1.0/) applies to the data made available in this article, unless otherwise stated. 


\section{Background \\ Rationale}

Dementia is fast becoming one of the greatest challenges to health and social care services in the UK. With an ageing population, the prevalence of dementia is growing rapidly with current UK estimates of 800,000 individuals living with dementia which is predicted to rise to over one million by 2021 [1]. Alongside the increasing prevalence come substantial costs. A recent report from the Alzheimer's Society states that the full societal cost of dementia has now reached an annual total of $£ 26.3$ billion, a figure comprising of costs for direct healthcare, social care (both private and publically funded) and the informal carers of people with dementia. This figure is greater than the combined costs of heart disease and stroke [2].

The complexity of needs that distinguishes this population must be addressed through a holistic and comprehensive approach to care. However, a number of recent government reports [3] and clinical guidance documents [4] have described the varying components of healthcare and social care responsible for the provision of dementia care as fragmented and poorly coordinated. These reports highlight the discrepancies between what current services are providing for the dementia population and what the research evidence suggests is effective high quality care, concluding that available services are suboptimal in standards and offer poor value for money. There is a frequent failure in individuals accessing recommended services and a lack in systematic help finding these services. Furthermore, when these services are accessed, there is an absence of continuity in long-term support [1], all of which leave capacity to improve the quality, efficiency and costs of dementia care.

One approach taken to improve the management of care provided to this population is the allocation of a health or social care professional to assume the responsibility of coordinating all aspects of care for the individual. These are organisational interventions which have been under development over recent decades and have been applied to a number of long-term conditions including dementia. The organisation and implementation of such interventions varies widely, but the primary focus is to develop a collaborative process of planning, facilitating and coordinating care and providing a proactive support base for both the patient and their informal caregiver/families. In the literature, the variations in these interventions have taken a number of different titles which are often used loosely and interchangeably including care/case management, collaborative care and care coordination.

The diversity and complexity present in these models of care has led to a patchy evidence base and a difficulty in defining the key components to measure their success. There have been a number of systematic reviews that have drawn mixed conclusions on the efficacy and impact of coordinating interventions [5-10]. Tam-Tham et al. [9] reported short-term effects of dementia case management on risk of institutionalisation, a result in agreement with Pimouguet et al. [8] who also reported a number of highquality trials demonstrating a positive impact of dementia case management in delaying institutionalisation. However, neither review found benefits on any further outcome measures. On the contrary, Somme et al. [7] reported on a number of randomised controlled trials (RCTs) demonstrating moderate effects on both clinical outcomes and resource utilisation.

Reviews of this nature face difficulty with the extent of heterogeneity across studies. This heterogeneity comes from a number of aspects including participant characteristics such as demographics, gender and disease severity; intervention characteristics such as case load, intensity and method of delivery; and contextual factors or from variation in outcome measures assessed [11]. With diversity in both the models of care and implementation methods, it becomes difficult to compare the impact of the interventions and what components are essential for its effectiveness. We will attempt to address these issues using a mixed studies review that is designed to be comprehensive, allowing the use of a vast range of research to address the complex nature of such health care interventions. Here, we outline the protocol of our review in accordance with the PRISMA-P [12] (2015) statement for preferred reporting items for systematic review and meta-analysis protocols (see Additional file 1).

\section{Objectives}

The aim of this review will be to identify the key components of community-based interventions coordinating care in dementia by asking the following questions:

1. What are the experiences, perceptions and views of stakeholders on community-based interventions coordinating care in dementia in terms of:

(i) the perceived key components of the interventions?

(ii) the helpful/unhelpful characteristics of the interventions?

(iii) the proposed mechanisms by which the interventions achieve their effect?

2. What are the effects of community-based interventions coordinating care in dementia on patient and carer outcomes?

3. Is there a relationship between the perceived key components of these interventions and the patient and carer outcomes?

To address these review questions, we will conduct an exploratory, sequential review design where the qualitative 
evidence will be synthesised first and will drive forward the secondary synthesis of quantitative evidence. With such a design, the quantitative results are mobilised to examine, confirm and generalise the qualitative findings [11]. Insights from both strands will be combined during the final stages of the review.

\section{Methods \\ Eligibility criteria \\ Types of studies}

We are conducting a mixed studies systematic review, and therefore, we will include studies of any design. We will consider inclusion of, but not limited to, the following study designs:

(1) Qualitative study design: qualitative studies that canvass the experiences, perceptions and views of stakeholders on dementia care coordination interventions.

(2) Quantitative study design: quantitative studies may include randomised controlled trials (RCTs) of intervention effectiveness or non-RCT designs (e.g. quasi-experimental, controlled/non-controlled before and after, cross-sectional and cohort studies).

(3) Mixed methods study design: we will also consider studies that have incorporated both strands in the methodological design.

No restrictions will be placed on study location. However, a flexible approach will be taken to the context of the study during the screening process, with particular care being paid to the global differences in health and social care systems. No restriction will be placed on language; however, a flexible approach will be taken here when considering the context of the study, in particular with regards to qualitative research, where study findings will be context specific and concepts can be lost in translation which may lead to a greater degree of inference. No restrictions will be applied to date of included studies.

\section{Types of participants}

We will include participants of any age and gender who are living in the community with a dementia diagnosis of any type (made by a clinician, such as a general practitioner (GP) or a hospital specialist, or made following standardised neuropsychiatric assessment) and their informal caregiver (if data are available). We will exclude studies that include people who self-define as having dementia (i.e. in the absence of a formal clinical diagnosis). The diagnostic uncertainty surrounding such participants is likely to increase the heterogeneity of our findings, and would definitely reduce the confidence and credibility with which we would be able to make future treatment recommendations for people with dementia, which is the main aim of our review. We will exclude studies that focus solely on interventions for the informal caregiver, which do not include an intervention focused on increased coordination of care or outcomes for the individual with dementia. Studies that do not focus on improving coordination of dementia care are beyond the scope of this review.

\section{Types of intervention}

We will include studies of interventions that are delivered in community settings to the specified population. The intervention must have an identified professional, key in the provision and management of care, who has a primary focus on care planning, coordination and proactive followup tailored to the needs of individual with dementia and/or informal caregivers. We will exclude any non-communitybased interventions such as those based in hospitals, care or residential homes. We will exclude studies that involve changes made to health care systems or application of guidelines alone.

\section{Comparator}

Many of the studies included will be of a qualitative or non-controlled design and therefore will not comprise a comparator group. Some quantitative studies reaching inclusion criteria, such as RCTs, will involve case-acceptable comparators such as treatment as usual, placebo control, waiting-list control or alternative dementia care interventions.

\section{Types of outcome measures}

We will examine a range of primary and secondary outcome measures. We will include studies that report on one or more health outcome measures that relate to the individual with dementia and/or informal caregiver, measures of resource use or process measures. These will have used standardised and validated measurement tools. Health outcome measures may include, but are not limited to, the following: depression and anxiety, carer burden, quality of life and cognition measures. Measures of resource use may include, but are not limited to, the following: institutionalisation, hospital admission rate and mean length of stay. Process measures may include, but are not limited to, the following: frequency of contact, case load intensity and professional background of case managers.

\section{Information sources}

(1) Database search: Electronic databases will be searched from data of inception to present, and the search syntax will be modified as appropriate for use in the following databases:

- MEDLINE (OvidSP)

- The Cochrane Library 
- EMBASE

- PsycINFO

(2) Citation search: Forward and backward citation searching will be conducted on included articles for further material. In the absence of required information, the first authors of studies will be contacted to request additional related material either unpublished or in press.

(3) Experts in the field: Experts in the field and corresponding authors of included studies will be contacted to gather further information.

(4) Grey literature search: To minimise the impact of publication bias, grey literature sources will be searched for unpublished material. Despite previous variations in the definition of grey literature, it has become commonly known as material that is not controlled by commercial publishers [13]. Examples of this type of material include government reports, policy documents, dissertation theses, book chapters and research reports. Searches for grey literature will be conducted in the Health Management Information Consortium (HMIC) and the Social Policy and Practice (SPP) databases, both of which will be accessed via OvidSP. HMIC is house to material including, but not limited to, Department of Health (DH) reports, Kings Fund data, and relevant health care books. SPP holds material including, but not limited to, government reports, health and social care services information and policy documents. The ProQuest database will be searched specifically for dissertations and theses. For additional information and material regarding RCTs, the International Clinical Trials Registry Platform (ICTRP) search portal will be used with an adjusted search strategy specified to the tools available on this platform.

\section{Search strategy}

A comprehensive search strategy has been developed and will use both controlled vocabulary unique to each database (e.g. MEDLINE Medical Subject Headings (MeSH) terms) and free-term texts. The strategy has been informed by discussions with experts in the field of dementia, complex interventions, systematic reviewing and by a prior scoping review identifying relevant keywords. An outline of the master search strategy for MEDLINE (OvidSP) can be found in Appendix 1. Our choice of electronic databases includes sources of grey literature to minimise the effects of publication bias. In addition, we will conduct a forward and backward citation searching of relevant papers and contact experts in the field for details of unpublished studies.

\section{Study records}

\section{Data management}

EndNote X7.0.2 software will be used to manage references throughout the review. Once the searches have been run, results will be exported to EndNote and any duplicates automatically identified will be removed. This process will be assisted by hand searching for duplicates.

\section{Screening}

Two independent reviewers will conduct an initial screening of titles and abstracts of identified papers for relevance in accordance with the outlined eligibility criteria. Full texts of potentially eligible studies will be retrieved, and a second screening will be conducted by two independent reviewers to produce a final set of papers to be included in the review. Disagreement at any stage will be resolved through discussion and referral to a third reviewer. A PRISMA diagram will be completed to show the flow of the screening process and number of records at each stage.

\section{Data extraction}

A bespoke data extraction sheet that has been developed in-house will be used. We anticipate that these will primarily be data-driven by studies found, but will be based on standardised forms for quantitative and qualitative extraction, and will be tailored to the characteristics of this review. The data extraction sheets will be piloted on six studies (three quantitative and three qualitative) identified at random from eligible studies and modified as required for use in this review. Once the final set of included studies has been established, data extraction sheets will be completed by two independent reviewers.

Data items to be extracted from qualitative studies will include, but not limited to, the following:

- General information (author(s), title, year of publication, journal of publication, country, and language).

- Study design (setting, recruitment, sample size, data collection, analysis).

- Participant characteristics (gender, age, stakeholder group).

- Data-text from 'results' and 'findings' from research reports. This data is likely to be presented in the form of themes and quotations.

Data items to be extracted from quantitative studies will include, but not limited to, the following:

- General information (author(s), title, year of publication, journal of publication, country, and language).

- Study design (setting, recruitment, eligibility, sample size, randomisation, data collection, analysis). 
- Participant characteristics (gender, age, diagnosis, comorbidities).

- Intervention (coordinating role, training, intensity, follow-up period, comparison groups).

- Outcome measures-Primary and secondary outcomes will be extracted from quantitative studies, including health outcome measures for both individual with dementia and caregiver and health process measures. This data is likely to include means, standard deviations and effect sizes.

We anticipate studies that have included mixed methodology will likely undergo two data extractions; although there will be some overlap, data will be extracted for the qualitative data sheet and for the quantitative data sheet. Where multiple publications have been produced from one study, data extraction will be conducted for each individual paper though the data will be considered as one study.

\section{Risk of bias}

Quality appraisal of included studies will be conducted by two independent reviewers using the Critical Appraisal Skills Programme (CASP) checklists [14]. CASP has a set of eight critical appraisal tools, and the tool selected for quality appraisal will be specific to the study design. We will consider the impact of the study quality on the findings of the review, possibly by comparing studies of high versus low quality, if sufficient suitable studies are identified. Assessing the quality of evidence in the review will also allow us to judge the strength of the conclusions and following recommendations.

\section{Data synthesis}

Synthesis of qualitative research will be dependent on the data. Where enough conceptual data is available, then it is anticipated that thematic synthesis, based on methods described by Thomas and Harden [15], will be used to address the initial review question and sub-review questions. In qualitative research, it can become difficult in defining what amounts to data, but here, our primary source of data will be, but not limited to, the text labelled as 'results' or 'findings' in study reports. Thematic synthesis will take form across three stages, beginning with an initial coding of primary data. Similarities and differences will then be sought within the codes allowing them to be organised into data-driven descriptive themes and permitting translation of concepts between studies. The final stage will involve the generation of analytic themes which will be driven by the review questions and their theoretical framework. However, if interpretive synthesis is not suitable for the data extracted, then a narrative synthesis approach will be taken.
Findings from the quantitative studies will be presented using narrative text and tabulation of the study characteristics, outcomes and risk of bias. Meta-analysis of RCTs will be considered if sufficient trials using comparable interventions and outcomes are identified. Outcomes from trials will be converted to the most appropriate standardised effect, which will then be pooled, using metaanalytic techniques [16]. Variation of study effect with differences in study, participant, setting and intervention characteristics will be estimated using the analogue of analysis of variance (ANOVA) or meta-regression, as appropriate. If meta-analysis is possible, publication bias will be assessed using Egger's test, forest plots and Duval's Trim and Fill methods.

Trials will only be considered for inclusion in the metaanalysis if the effects of the coordinating intervention can be isolated from that of the control. Acceptable designs for a trial to be incorporated into the meta-analysis include, for example:

(1) Coordinating intervention plus treatment as usual versus treatment as usual

(2) Coordinating intervention plus alternate intervention versus alternate intervention

Trials will not be included in the meta-analysis where effects of target intervention cannot be isolated, for example, target intervention versus alternate intervention. Studies with this later design will be described in the narrative section and tables but will be excluded from meta-analysis.

Once analysis of data is complete, the final stage of the review will merge the two strands of data through a narrative synthesis to conclude the findings and propose how the project will progress to develop a community-based intervention for coordinating care in dementia.

\section{Discussion}

This mixed studies review will provide a detailed account of the evidence base underpinning interventions that coordinate healthcare for people with dementia. Synthesis of study findings will isolate components of interventions perceived as important by identified stakeholders and those that are effective at improving outcomes. Our interpretation of the synthesis of study findings will take account of limitations in studies identified and any limitations in our own review methodology.

To our knowledge, this is the first review to address associations between perspectives of stakeholders and the effectiveness of interventions. The mixed studies sequential design is required for the multiple layers of review questions and allows us to gain both breadth and depth in covering evidence 
from multiple sources. However, this review design is demanding in time and resources. The use of multiple reviewers will help reduce time burden and minimise the risk of bias. We will discuss how the findings of our review compare and contrast with findings from other reviews of effectiveness of coordinating interventions in dementia [5-11].

The findings of this review will be used to develop a theoretical model of an intervention coordinating dementia care for evaluation in future research. We will also discuss how the evidence supporting care coordinating interventions for people with dementia will be useful to both policy makers and health care providers, informing the future design of services and specific interventions for people with dementia.

\section{Appendix}

Appendix 1: Master Search Strategy in MEDLINE (OvidSP) Database: Ovid MEDLINE(R) In-Process \& Other NonIndexed Citations and Ovid MEDLINE(R) <1946 to Present>

Search strategy:

1 exp Dementia/

2 dement*.mp.

3 alzheimer*.mp.

4 (presenile/ or senile.mp.) and dement*.mp. [mp = title, abstract, original title, name of substance word, subject heading word, keyword heading word, protocol supplementary concept word, rare disease supplementary concept word, unique identifier]

5 *Delirium, Dementia, Amnestic, Cognitive Disorders/

6 *cognition disorders/ or *mild cognitive impairment/

71 or 2 or 3 or 4 or 5 or 6

8 Case Management/

9 collaborative care.mp.

10 case manag*.ti,ab.

11 care manag*.ti,ab.

12 (care adj2 coordinat*).ti,ab.

13 (case adj2 coordinat*).ti,ab.

14 service coordinat*.ti,ab.

15 care consult".ti,ab.

16 case consult".ti,ab.

17 (care adj2 facilitat*).ti,ab.

18 shared care.ti,ab.

19 (coordinat* adj2 care).ti,ab.

20 admiral nursing.mp.

21 *disease management/

228 or 9 or 10 or 11 or 12 or 13 or 14 or 15 or 16 or 17 or 18 or 19 or 20 or 21 (33785)

237 and 22

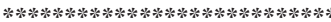

[mp=title, abstract, original title, name of substance word, subject heading word, keyword heading word, protocol supplementary concept word, rare disease supplementary concept word, unique identifier]

[ti,ab=title \& abstract]

\section{Additional file}

Additional file 1: PRISMA-P 2015 Checklist. Items that have been reported in the protocol from the required PRISMA-P checklist. (DOC 17 kb)

\section{Abbreviations}

ANOVA: analysis of variance; CASP: Critical Appraisal Skills Programme; DH: Department of Health; GP: general practitioner; HMIC: Health Management Information Consortium; ICTRP: International Clinical Trials Registry Platform; MeSH: Medical Subject Headings; NIHR: National Institute for Health Research; RCT: randomised controlled trial; SPP: Social Policy and Practice.

\section{Competing interests}

There are no competing interests to declare.

\section{Authors' contributions}

$A B, C D, D R$ and $R M$ conceived and designed the systematic review. AB wrote the initial draft of the review protocol, which was then revised by $C D$ and $D R$. AB registered the review protocol on the PROSPERO database. The final manuscript was read and approved by all authors.

\section{Acknowledgements}

This research was funded by the National Institute for Health Research (NIHR) Collaboration for Leadership in Applied Health Research and Care South West Peninsula. The views expressed are those of the author(s) and not necessarily those of the NHS, the NIHR or the Department of Health. Authors would like to acknowledge the protocol contributions from Darren Moore through use of the NIHR CLAHRC for the South West Peninsula Evidence Synthesis Research Clinics.

Received: 28 August 2015 Accepted: 23 October 2015

Published online: 07 November 2015

\section{References}

1. Prince $M$, Knapp M, Guerchet M, McCrone P, Prina M, Comas-Herrera A, Wittenberg R, Adelaja B, Hu B, King D, Rehill A, Salimkumar D. Dementia UK: update (second edition). Alzheimer's Society; 2014.

2. Spotlight on Dementia Care. A Health Foundation improvement report. London: The Health Foundation; 2011.

3. Improving dementia services in England_an interim report. London; National Audit Office: 2010.

4. Dementia quality standards. Manchester; National Institute for Health and Care Excellence: 2010.

5. Khanassov V, Vedel I, Pluye P. Case management for dementia in primary health care: a systematic mixed studies review based on the diffusion of innovation model. Clin Interv Aging. 2014;11(9):915-28.

6. Khanassov V, Vedel I, Pluye P. Barriers to implementation of case management for patients with dementia: a systematic mixed studies review. Ann Fam Med. 2014;12(5):456-65.

7. Somme D, Trouve H, Drame M, Gagnon D, Couturier Y, Saint-Jean O. Analysis of case management programs for patients with dementia: a systematic review. Alzheimers Dement. 2012;8(5):426-36.

8. Pimouguet C, Lavaud T, Dartigues JF, Helmer C. Dementia case management effectiveness on health care costs and resources utilization: a systematic review of randomized controlled trials. J Nutr Health Aging. 2010;14(8):669-76.

9. Tam-Tham H, Cepoiu-Martin M, Ronksley PE, Maxwell CJ, Hemmelgarn BR. Dementia case management and risk to long-term care placement: a systematic review and meta-analysis. Int J Geriatr Psychiatry. 2012;28(9):889-902.

10. Reilly S, Miranda-Castillo C, Malouf R, Hoe J, Toot S, Challis D, Orrell M. Case management approaches to home support for people with dementia (Review). The Cochrane Database of Systematic Reviews. 2015; doi: 10.1002/14651858.CD008345. 
11. Hannes K. Building a case for mixed-methods reviews. In: Richards DA Hallberg IR, editors. Complex interventions in health: an overview of research methods. Oxton: Routledge; 2015. p. 88-95.

12. Moher D, Shamseer L, Clarke M, Ghersi D, Liberati A, Petticrew M, Shekelle P, Stewart LA, PRISMA-P Group. Preferred reporting items for systematic review and meta_-analysis protocols (PRISMA-P) 2015 statement. Systematic Reviews. 2015; doi: 10.1186/2046-4053-4-1.

13. Hopewell S, McDonald S, Clarke M, Egger M. Grey literature in meta-analysis of randomized trials of health care interventions. The Cochrane Database of Systematic Reviews. 2007; doi:10.1002/14651858.MR000010.pub3.

14. Critical Appraisal Skills Programme (CASP). CASP Checklist. http://www.caspuk.net/\#!lcasp-tools-checklists/c18f8 (2014). Accessed 21 April 2015.

15. Thomas J, Harden A. Methods for the thematic synthesis of qualitative research in systematic reviews. BMC Medical Research Methodology. 2008; doi:10.1186/1471-2288-8-45.

16. Lipsey MW, Wilson DB. Practical meta-analysis (applied social research methods series, Vol. 49). Thousand Oaks, CA: Sage; 2001.

\section{Submit your next manuscript to BioMed Central and take full advantage of:}

- Convenient online submission

- Thorough peer review

- No space constraints or color figure charges

- Immediate publication on acceptance

- Inclusion in PubMed, CAS, Scopus and Google Scholar

- Research which is freely available for redistribution 